\title{
Expression of Death Receptor 3 (DR3) on Circulating CD4 Positive Lymphocytes on Patients with Vitiligo
}

\author{
A.M.Al-Refahie ${ }^{1}$, A.I.Mustafa ${ }^{1}$, R.H.Abdel Sattar ${ }^{2}$ and G.S.Abd El-Aziz ${ }^{1}$ \\ ${ }^{1}$ Dermatology, Venereology and Andrology Dept., Faculty of Medicine, Benha Univ., Benha, Egypt \\ ${ }^{2}$ Clinical and Chemical Pathology Dept., Faculty of Medicine, Benha Univ., Benha, Egypt
}

E-Mail: Dr-Haloo@yahoo.com

\begin{abstract}
Vitiligo is the most common depigmenting skin disorder with an estimated prevalence of $0.5-2 \%$ of the population in both adults and children worldwide. Death receptor 3 is a typical member of TNFRSF which play a role in apoptotic signalling and differentiation. DR3 is considered the primary activating receptor of TL1A and is predominantly expressed on leucocytes. DR3 expression is significantly upregulated on various activated cells in the immune system, including T lymphocytes, B lymphocytes, natural killer T (NKT) cells, dendritic cells and macrophages. The aim of this study was to study the expression of death receptor 3 (DR3) on CD4 positive lymphocytes on patients with vitiligo . This study was a case control study that included 50 patients with vitiligo in addition to 25 apparently healthy individuals of matched age and sex as a control group. All patients were recruited from the outpatient clinic of Dermatology, Venereology and Andrology Department of Benha University Hospitals. DR3 showed fair AUC (AUC=0.794). At cut off value of 23.8, sensitivity was72\%, specificity was $80 \%$, PPV was $87.8 \%$, NPV was $58.8 \%$, and accuracy was $74.7 \%$. DR3 expression level was significantly higher in vitiligo cases when compared to control group. DR3 expression level was significantly correlated with BSA \%. DR3 expression level was suggested to be independent predictor of vitiligo susceptibility and Extent.
\end{abstract}

Keywords: Death Receptor 3, DR3, CD4,Vitiligo.

\section{Introduction}

Vitiligo is an acquired cutaneous disorder of pigmentation, with a $0.5-2 \%$ incidence worldwide that affects males and females alike. It is characterized by progressive depigmentation and the appearance of white cutaneous macules, usually with no other clinical symptoms. Clinically it can present as a non-segmental variant, with bilateral and generalized distribution (the most common form) or as asegmental variant, involving a single body region. epigmentation is due to the loss of functioning melanocytes [1].

Numerous hypotheses about the etiology of vitiligo had been found however, none of them was proven. One way to understand the etiology was to determine the mechanism by which melanocytes were destroyed. Two known mechanisms for this cell loss were found, necrosis and apoptosis. The histology of vitiligo strongly suggests apoptosis rather than necrosis as the mechanism for melanocyte destruction [2].

Death receptors is a part of tumor necrosis factor (TNF) receptor gene super family, which is defined by similar, cysteine-rich extracellular domains. Death receptors contain a homologous cytoplasmic sequence termed the "death domain". Death domains typically enable death receptors to engage the cell's apoptotic machinery, but in some instances they mediate functions that are distinct from or even counteract apoptosis. Some molecules that transmit signals from death receptors contain death domains themselves [3].

Death receptors (DRs) include TNF-areceptor 1 (TNF-R1), Fas, DR3, DR4 (TNF-related apoptosis inducing ligand-R1; TRAIL-R1), DR5 (TRAIL-R2), and DR6, whereas death ligands include TNF- $\alpha$, Fas-ligand (FasL), Apo3-ligand, TRAIL, and lymphotoxin. DR3 or TNFRSF25 was reported simultaneously by a number of groups as a tumor necrosis factor receptor superfamily (TNFRSF) member with an intracellular, apoptosisinducing death domain and was ascribed a variety of names -Apo3, LARD, TR3, TRAMP and WSL-1. In recent years, DR3 has emerged as a major regulator of inflammatory and autoimmune disease. Its only known TNFSF ligand is TNF-like protein 1A (TL1A) [4].

The TNF family of receptors and ligands is a major regulator not only of conventional CD4+ and CD8+ $\mathrm{T}$ cells but also of Tregs (Regulatory $\mathrm{T}$ cells) Therefore, not surprisingly, DR3 was initially identified as a T-cell co stimulatory molecule [5].

The interaction of TL1A and its receptor DR3 has been found to be involved in the pathogenesis of several inflammatory and autoimmune diseases, such as rheumatoid arthritis, inflammatory bowel disease, psoriasis and multiple sclerosis [6].

The role and mechanism of DR3 in a physiopathologic context remains unclear. DR3 induces cellular apoptosis and proliferation via activation of the caspase apoptotic cascade or the transcription factor nuclear factor- $\kappa \mathrm{B}(\mathrm{NF}-\kappa \mathrm{B})$, stress-activated protein kinase (SAPK), c-Jun N-terminal protein kinase (JNK) and p38 mitogen-activated protein kinase (MAPK). DR3 contains a cytoplasmic death domain (DD), sharing certain similarity with TNFR1 and CD95. The signaling pathways induced by these receptors are similar and rely on the oligomerization of the receptors. Upon binding with ligands, recruitment of death domain proteins,, through homophilic interaction of their death domains subsequently activate the caspase apoptotic cascade or the NF- $\kappa$ B pathway [7].

The aim of this work was to study the expression of death receptor 3 (DR3) on CD4 positive lymphocytes on patients with vitiligo. 


\section{Patients and methods}

This study was a case-control study. This study included 50 patients with vitiligo. In addition to 25 apparently healthy individuals of matched age and sex as a control group. All patients were recruited from the outpatient clinic of dermatology and andrology department.

The study was approved by the ethics committee of research involving human subjects of Benha faculty of medicine. Informed consent was obtained from each individual before being enrolled in the study

\subsection{Inclusion criteria}

- The study included patients suffering from different clinical types and degrees of severity of vitiligo who were diagnosed clinically and the diagnosis was confirmed by woods light .

- The age of patients ranged (18-45) years and both sexes were involved.

\subsection{Exclusion Criteria}

The following were excluded from the study :

- Patients with other autoimmune diseases e.g: rheumatoid arthritis, inflammatory bowel disease and systemic lupus erythematous.

- Patients with cancer colon, cancer prostate and cancer breast.

- All patients were subjected to full history taking, complete clinical examination, and laboratory investigations. Assessment of DR3 expression on circulating CD4 positive lymphocytes on both patients with alopecia areata and control group by flow cytometric analysis.

\subsection{Statistical analysis}

The collected data was revised, coded, tabulated and introduced to a PC using Statistical package for Social Science (IBM Corp. Released 2017. IBM SPSS Statistics for Windows, Version 25.0. Armonk, NY: IBM Corp.). Data were presented and suitable analysis was done according to the type of data obtained for each parameter. Descriptive statistics: Mean Standard deviation $( \pm$ SD) for numerical data. Frequency and percentage of non-numerical data. Shapiro test was done to test the normality of data distribution. Analytical statistics: Student T Test was used to assess the statistical significance of the difference between two study group means. For the comparison of the three groups' means, one way analysis of variance (ANOVA) was used. ChiSquare test was used to examine the relationship between two qualitative variables. Fisher's exact test: was used to examine the relationship between two qualitative variables when the expected count is less than 5 in more than $20 \%$ of cells. Correlation analysis: To assess the strength of association between two quantitative variables. The correlation coefficient defines the strength and direction of the linear relationship between two variables. The ROC Curve (receiver operating characteristic) provides a useful way to evaluate the sensitivity and specificity for quantitative diagnostic measures that categorize cases into one of two groups. The optimum cut off point was defined as that which maximized the AUC value. The area under the ROC curve (AUC) results were considered excellent for AUC values between 0.9-1, good for AUC values between 0.80.9, fair for AUC values between 0.7-0.8, poor for AUC values between 0.6-0.7 and failed for AUC values between 0.5-0.6. Regression analysis: Logistic and ordinal regression analyses were used for prediction of risk factors, using generalized linear models.

\section{Results}

The present study included 50 vitiligo patients and 25 healthy control subjects. Studied patients and control groups were age and gender matched $(\mathrm{p}>0.05$ for each) Table (1).

Table (1) Comparison of demographic databetween cases and control groups.

\begin{tabular}{lcccccc}
\hline Variable & & \multicolumn{2}{c}{ Control } & \multicolumn{2}{c}{$\begin{array}{c}\text { Vitiligo } \\
\text { N=50 }\end{array}$} & p \\
& & \multicolumn{2}{c}{$\mathbf{N = 2 5}$} & 38.2 & 10.8 & 0.537 \\
Age (years) & mean \pm SD & 39.8 & 9.9 & 12 & $24 \%$ & 0.460 \\
Males & $\mathrm{N}, \%$ & 8 & $32 \%$ & 38 & $76 \%$ & \\
Females & $\mathrm{N}, \%$ & 17 & $68 \%$ & 38 & \\
\hline
\end{tabular}

The expression of death receptor 3 (DR3) on CD4 positive lymphocytes in vitiligo group showed significant higher expression level $(\mathrm{p}<0.001)$ Fig $(1)$.

Receiver operating characteristic (ROC) curve of DR3 expression level were conducted for discrimination between vitiligo and control groups. DR3showed fair AUC (AUC=0.794). At cut off value of 23.8, sensitivity was $72 \%$, specificity was $80 \%$, PPV was $87.8 \%$, NPV was $58.8 \%$, and accuracy was $74.7 \%$. Fig (2).

No significant associations were found in DR3 expression level with gender, family history or course disease in vitiligo group ( $p>0.05$ for each) Table (2).
DR3 expression level showed significant positive correlation with disease extent, but not with age, age of onset or duration in vitiligo group Table (3).

Linear regression analysis was conducted for prediction the extent of vitiligo (BSA \%) using age, gender, family history, onset, course, and duration showed that DR3 expression level as confounders. Higher DR3was suggested to be independent risk predictor for vitiligo extent Table (4). 


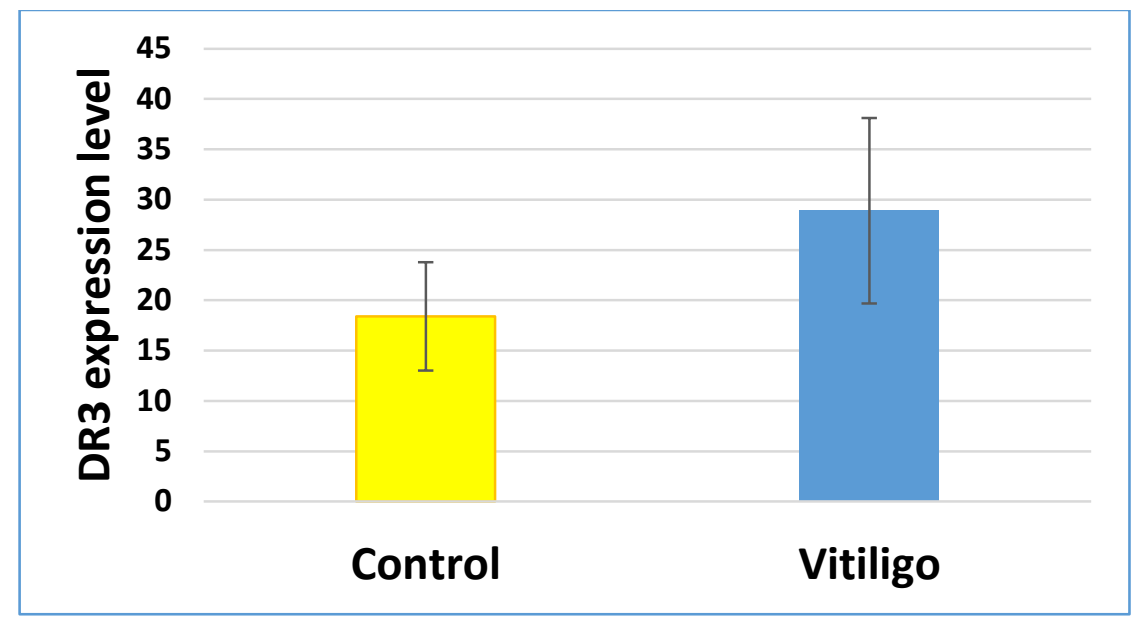

Fig (1) Bar chart for DR3 expression level in studied groups. Bar represents mean, while error bar represents standard deviation.

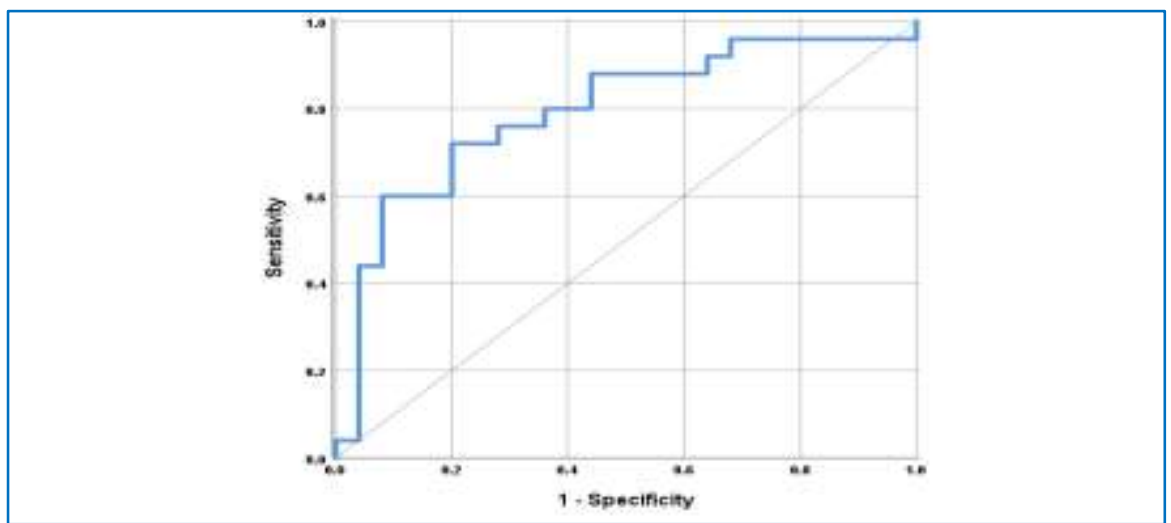

Fig (2) ROC curve of DR3 expression level for discrimination between vitiligo and control groups.

Table (2) Comparison of DR3 expression level according to studied parameters in vitiligo group.

\begin{tabular}{lcccccc}
\hline Variable & & \multicolumn{4}{c}{ DR3 expression level } & \multirow{2}{*}{$\boldsymbol{P}$} \\
\cline { 3 - 6 } & & $\mathbf{N}$ & mean & \pm & SD & \\
Gender & Male & 12 & 25.1 & \pm & 7.6 & 0.123 \\
\multirow{3}{*}{ Family history } & Female & 38 & 29.8 & \pm & 8.6 & \\
& Negative & 40 & 28.0 & \pm & 9.3 & 0.191 \\
Course & Positive & 10 & 32.7 & \pm & 9.9 & \\
& Stationary & 18 & 31.4 & \pm & 6.6 & 0.119 \\
\hline
\end{tabular}

$\mathrm{SD}$, standard deviation; student $\mathrm{t}$ test and ANOVA was used for numerical parameters.

Table (3) Correlations of DR3 with age, age of onset, duration and extent in vitiligo group.

\begin{tabular}{lcc}
\hline Variable & \multicolumn{2}{c}{ DR3 } \\
\cline { 2 - 3 } & $\mathbf{r}$ & $\mathbf{p}$ \\
\hline Age & -0.104 & 0.504 \\
Age of onset & 0.198 & 0.236 \\
Duration & 0.071 & 0.626 \\
Extent & 0.992 & $<0.001$ \\
\hline
\end{tabular}


r, Pearson's correlation coefficient

Table (4) Regression analysis for prediction of factors affecting Extent of vitiligo (BSA \%).

\begin{tabular}{lcc}
\hline & $\boldsymbol{\beta}$ & $\mathbf{P}$ \\
\hline Age & -0.073 & 0.146 \\
Gender & 0.554 & 0.662 \\
Family history & 1.872 & 0.162 \\
onset & 0.060 & 0.181 \\
Duration & 0.004 & 0.957 \\
Course & 2.507 & 0.122 \\
DR3 & 0.255 & $<0.001$ \\
\hline
\end{tabular}

\section{Discussion}

Results of this study showed that DR3 expression level was significantly higher in vitiligo cases when compared to control group, as well as it was significantly correlated with vitiligo BSA\%. Higher level of DR3was suggested being independent risk predictor for vitiligo development. In addition, it was suggested to be independent risk predictor for vitiligo extent.

The role of DR3 in AA pathogenesis can be explained by the following findings

Cellular immunity is one of important factor in the development of vitiligo where $\mathrm{CD} 8+\mathrm{T}$ cell predominant infiltrates have been found in perilesional skin, and CD8+ cytotoxic $\mathrm{T}$ cells have been identified adjacent to disappearing melanocytes (van den Wijngaard et al., 2000). In addition, it was found that DR3 expression is restricted on CD4+ and CD8+ $\mathrm{T}$ cells, natural killers (NK) cells, regulatory $\mathrm{T}$ cells and DR3 expression is increased after immunological activation of these cells [8].

The TNF family of receptors and ligand is a major regulator not only of conventional $\mathrm{CD} 4+$ and $\mathrm{CD} 8+\mathrm{T}$ cells but also of Tregs (Regulatory $\mathrm{T}$ cells) Therefore, not surprisingly, DR3 was initially identified as a T-cell co stimulatory molecule [9]. and a role for the TL1ADR3 system in the development of inflammation- driven pathologies such as autoimmunity, inflammatory bowel disease, rheumatoid arthritis, and psoriasis has been proposed [10].

DR3 expression is significantly up regulated on various activated cells in the immune system, including T lymphocytes, B lymphocytes, natural killer T (NKT) cells, dendritic cells and macrophages. Previous studies have indicated that DR3 is a very powerful lymphocyte co stimulatory, and DR3 signalling amplifies a series of immune responses, culminating in the activation of $\mathrm{T}$ lymphocytes, and initiates the secretion of pro inflammatory cytokines [11].

It is established that TL1A/DR3 signalling results in a generalized, non-specific stimulation of all major $\mathrm{T}$ cell effector pathways, for example, Th1, Th2 and Th17, which have been shown to play a pivotal role in the pathogenesis of psoriasis [12]. In addition it was found that Th1, Th2, and more recently Th17 type cytokines have been significantly quantified in the sera and skin of patients with vitiligo [13]. Where they play an important role in its pathogenesis.
Vitiligo Biopsy results have shown that $\mathrm{T}$ cells separated from lesional skin were significantly enriched for recognizing specific melanocytes antigens, and when they migrated to the melanocytes in unaffected normal skin, they induced apoptosis of melanocytes. However, perilesional $\mathrm{T}$ cells did not in $\neg$ duce apoptosis in lesional skin, for loss of melanocytes, there $\neg$ fore suggesting melanocyte-specific cytotoxic activity of CD8+ T cells [14].

By contrast, CD8+ T cell-depleted perilesional $\mathrm{T}$ cells did not have the capacity to induce cytotoxicity and apoptosis of melanocytes cells [14].

In addition, the following may illustrate the role of DR3 in vitiligo apoptosis.TL1A-DR3 signalling induces both NF- $\kappa \mathrm{B}$ activation and apoptosis in vitro. TL1ADR3 interaction induces the formation of signalling complexes containing TRADD, TRAF2, and RIP and activates the NF- $\kappa \mathrm{B}$ and MAPK pathways (ERK, p38, and JNK). On the other hand, DR3 overexpression in embryonic cells also induces FADD- and caspase-8dependent apoptosis. [15].

The role of DR3 in vitiligo pathogenesis can be explained and support the research results by explaining and analysis the following findings of previous similer pathogenesis

Cumulative evidence from studies suggests the contribution of DR3 in in many immune processes and in various autoimmune diseases sharing pathogenic pathways similar to vitiligo. For example, elevated serum TL1A acts on mononuclear cells through increased DR3 expression on CD8+ lymphocytes and CD14+ lymphocytes in the peripheral blood of patients with psoriasis vulgaris. TL1A could act in synergy with IL-23 to stimulate PBMCs from patients with PV to produce IL-17 that has role in pathogenesis in psoriasis [16].

Death receptor 3 (DR3) has been found to be involved in the pathogenesis of several inflammatory and autoimmune diseases, such as rheumatoid arthritis, inflammatory bowel disease, allergic lung inflammation ,psoriasis, multiple sclerosis and diabetic retinopathy [17]. Another study showed that both protein expressions and mRNA transcripts of TL1A and DR3 were increased in psoriatic lesions [10].

TL1A/DR3 signaling exerts pleiotropic effects on lymphocyte homeostasis, cell proliferation, activation, function, and differentiation, mediating the balance between inflammatory and Treg responses. Additional 
data supporting the role of DR3 in Treg functionality consist in the observation that treatment with $4 \mathrm{C} 12$, an agonistic antibody to DR3, induces selective expansion of Tregs and reduces activation of conventional T cells in an allergic lung mouse model, in cardiac allograft [18].

\section{Conclusion}

DR3 expression level was significantly higher in vitiligo cases when compared to control group. DR3 expression level was significantly correlated with BSA $\%$. DR3 expression level was suggested to be independent predictor of vitiligo susceptibility and Extent.

\section{References}

[1] M. Rashighi and J. E. Harris, "Vitiligo pathogenesis and emerging treatments," Dermatol. Clin., Vol.35(2), PP.257-265, 2017.

[2] C. L. Huang, J. J. Nordlund, and R. Boissy, "Vitiligo," Am. J. Clin. Dermatol., Vol.3 (5), PP.301-308, 2002.

[3] H. Wajant, "Death receptors.," Essays Biochem., Vol.39(4), P. 53, 2003.

[4] H. Walczak, "Death receptor-ligand systems in cancer, cell death, and inflammation," Cold Spring Harb. Perspect. Biol., Vol.5(5), P. a008698, 2013.

[5] M. Croft, "The role of TNF superfamily members in T-cell function and diseases," Nat. Rev. Immunol., Vol.9(4), PP.271-285, 2009.

[6] Y. Aiba and M. Nakamura, "The role of TL1A and DR3 in autoimmune and inflammatory diseases," Mediators Inflamm., Vol.2013(2), PP.456, 2013.

[7] Z. Ge, A. J. Sanders, L. Ye, and W. G. Jiang, "Aberrant expression and function of death receptor3 and death decoy receptor-3 in human cancer," Exp. Ther. Med., Vol.2(2), PP.167-172, 2011.

[8] R. Watanabe et al., "Human skin is protected by four functionally and phenotypically discrete populations of resident and recirculating memory $\mathrm{T}$ cells," Sci. Transl. Med., Vol.7(279), PP.279ra39279ra39, 2015.

[9] T.-S. Migone et al., "TL1A is a TNF-like ligand for
DR3 and TR6/DcR3 and functions as a T cell costimulator," Immunity, Vol.16(3), PP.479-492, 2002.

[10]G. Bamias et al., "Upregulation and nuclear localization of TNF-like Cytokine 1A (TL1A) and its receptors DR3 and DcR3 in psoriatic skin lesions," Exp. Dermatol., Vol.20(9), PP.725-731, 2011.

[11] K. Reichwald, T. Z. Jørgensen, P. Tougaard, and S. Skov, "TL1A induces TCR independent IL-6 and TNF- $\alpha$ production and growth of PLZF+ leukocytes," PLoS One, Vol.9(1), P. e85793, 2014.

[12]L.-G. Jia et al., "A novel role for TL1A/DR3 in protection against intestinal injury and infection," $J$. Immunol., Vol.197(1), PP.377-386, 2016.

[13]Z. A. Taher, G. Lauzon, S. Maguiness, and M. T. Dytoc, "Analysis of interleukin-10 levels in lesions of vitiligo following treatment with topical tacrolimus," Br. J. Dermatol., Vol.161(3), PP.654659, 2009.

[14] J. G. Van Den Boorn et al., "Autoimmune destruction of skin melanocytes by perilesional $\mathrm{T}$ cells from vitiligo patients," J. Invest. Dermatol., Vol.129(9), PP.2220-2232, 2009.

[15] S. Madireddi et al., "Regulatory T Cell-Mediated Suppression of Inflammation Induced by DR3 Signaling Is Dependent on Galectin-9," J. Immunol., Vol.199(8), PP.2721-2728, 2017.

[16]L. Li et al., "Expression of death receptor 3 (DR3) on peripheral blood mononuclear cells of patients with psoriasis vulgaris," Postgrad. Med. J., Vol.94(1116), PP.551-555, 2018.

[17]Z. Zhang et al., "Changes in TL1A levels and associated cytokines during pathogenesis of diabetic retinopathy," Mol. Med. Rep., Vol.15(2), PP.573580, 2017.

[18] D. Wolf et al., "Tregs expanded in vivo by TNFRSF25 agonists promote cardiac allograft survival," Transplantation, Vol.94( 6), PP.569-574, 2012. 